\title{
COSMIC RAYS AND EARTH'S CLIMATE
}

\author{
HENRIK SVENSMARK \\ Danish Space Research Institute, DK-2100 Copenhagen Ø, Denmark
}

Submitted: 13 August 1999;

\begin{abstract}
During the last solar cycle Earth's cloud cover underwent a modulation in phase with the cosmic ray flux. Assuming that there is a causal relationship between the two, it is expected and found that Earth's temperature follows more closely decade variations in cosmic ray flux than other solar activity parameters. If the relationship is real the state of the Heliosphere affects Earth's climate.
\end{abstract}

\section{Introduction}

The physical cause of climate variability is not know in detail. There are several physical factors that are believed to influence Earth's climate. For example: (1) Orbital changes in Earth's motion around the sun is believed to cause iceages. (2) Internal variability in the climate system, e.g. changes in atmospheric and ocean circulation. (3) Large volcanic eruptions, which are known to cause a sudden cooling lasting 2-3 years. A period with high volcanic activity could potentially lead to a cooling of Earth. (4) Changes in concentration of greenhouse gases. Due to burning of fossil fuel during the last 100 years there has been an increase in atmospheric $\mathrm{CO}_{2}$ concentration from about 280 to $365 \mathrm{ppm}$. Because $\mathrm{CO}_{2}$ is a greenhouse gas that traps outgoing long wave radiation, and that the surface temperature has increased by approximately $0.7^{\circ} \mathrm{C}$ during the last 100 years, there is a worry that this increase is leading to a warmer climate. (5) Changes in solar activity, which will be discussed further in this paper. The relative importance of the above different influences is not know very well.

It is obvious from historical records that the Sun has played an important role in the climate of the Earth. For more than a hundred years there have been reports of an apparent connection between solar activity and Earth's climate (Eddy, 1976; Herman and Goldberg, 1978). William Herschel, a well known scientist in London suggested in 1801 that the price of wheat was directly controlled by the number of sunspots, based on his observation that less rain fell when there was few sunspots. Since then many reports have indicated a link between solar activity and climate. Solar activity is known for a long time back in time through a history of atmospheric isotope levels produced by galactic cosmic rays (see also the article of J. Beer in this book). Such records reveal a striking qualitative agreement between cold and warm climatic periods and low and high solar activity during the last 10,000 years (Eddy, 1976). Figure 1 shows the variation in ${ }^{14} \mathrm{C}$ concentration during the last millennium. From year 1000-1300 AD solar activity was very high, which coincided with the warm medieval period. It was during this period that the Vikings settled in Greenland. Solar activity decreased considerably after 1300 AD and a long cold period followed, called the little ice age. This climatic shift was

Space Science Reviews 93: 155-166, 2000.

(C) 2000 Kluwer Academic Publishers. Printed in the Netherlands. 


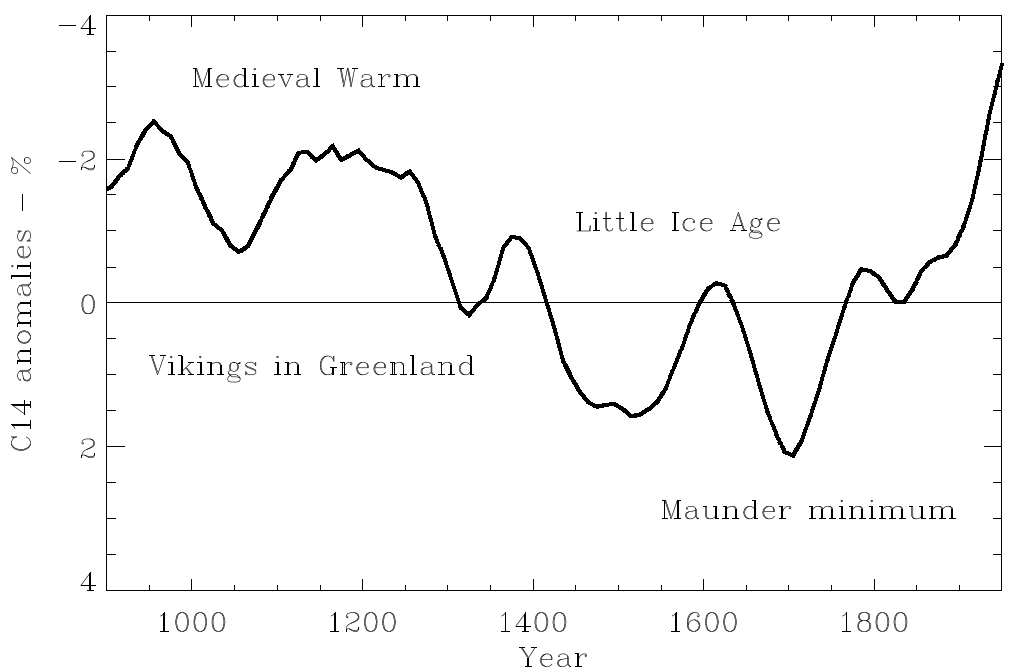

Figure 1. Change in ${ }^{14} \mathrm{C}$ concentration during the last 1,000 years. The variation in the ${ }^{14} \mathrm{C}$ concentration is dominated by changes in solar activity. When solar is high the production of $\mathrm{C}^{14}$ is low, due to the shielding effect of the solar wind against cosmic rays. Note that the axis for the ${ }^{14} \mathrm{C}$ concentration has been reversed. The Maunder minimum refers to the period 1645-1715 when very few sunspots where observed on the sun. In this period the concentration of ${ }^{14} \mathrm{C}$ was higher in agreement with a low solar activity.

a disaster for the Vikings. The Little Ice Age lasted until the middle of the last century. During this century solar activity has again increased and is at its highest level the past 600 years.

It is not only proxy data that show an apparent agreement with solar activity. An indication of a link between long term variations in solar activity and Earth's temperature was found by Friis-Christensen and Lassen (1991) and Lassen and Friis-Christensen (1995). They showed that an empirically constructed measure of solar activity, the filtered solar cycle length, closely matched variations in northern hemispheric temperature during the last 400 years. Another interesting example of a solar influence was discovered by Labitzke and van Loon (1993). They showed that the height of the pressure surfaces in the lower stratosphere varies in phase with solar activity and has done so through the last four solar cycles.

The most obvious and direct way solar activity could affect Earth's climate would be via changes in solar irradiance. But the steadiness of the sun has been established by satellite measurements of solar irradiance during the last 20 years. It is found that the variations are small $\left(0.1 \% \approx 0.3 \mathrm{~W} / \mathrm{m}^{2}\right.$ during a solar cycle), although this is not completely negligible, it is too small to explain the observed temperature changes (Lean et al., 1995). Apart from a direct influence of solar irradiance variations, there have been speculations on how small changes in solar activity can be amplified in the Earth's atmosphere. One idea is related to the fact that during a solar cycle, changes in the UV radiation of the solar spectrum are of the order of $10 \%$. Due to the importance of UV in the formation of ozone it 
has been suggested that the resulting heating in the stratosphere is dynamically transported down into the troposphere (Haigh, 1996; Shindell et al., 1999).

Another suggestion involves galactic cosmic rays (GCR). GCR consists of very energetic particles (mainly protons) that are accelerated in stellar processes in our galaxy. Some of them enter Earth's atmosphere where nuclear processes take place and produce secondary particles that can penetrate still deeper into the atmosphere (Lal et al., 1967). Ionisation in the atmosphere below $35 \mathrm{~km}$ is almost exclusively produced by GCR, except for the lowest $1 \mathrm{~km}$ over land where radioactive gases are the main cause of ionisation. Ionisation by GCR is the variable of the lower atmosphere subject to the largest solar cycle modulation(Ney, 1959). The ionisation variation could potentially influence optical transparency of the atmosphere, by either a change in aerosol formation and/or an influence on the transition between the different phases of water (Ney, 1959; Dickinson, 1975; Pudovkin and Raspopov, 1992; Pudovkin and Veretenenko, 1992; Tinsley, 1996; Svensmark et al., 1997; Svensmark et al., 1998).

In the following it will be shown that Earth's cloud cover, obtained from satellites, within the last solar cycle follows variations in GCR more closely than other solar activity parameters. Further it will be shown that long term variation in solar activities given by GCR reflects variations in Earth's temperature during the period (1937-1994) based on direct measurement of cosmic ray flux. Finally an indication of a GCR influence on temperatures during the Maunder minimum is presented.

\section{Cosmic Rays and Earth's Climate}

\subsection{COSMic Rays And Clouds}

Recently it was found that the Earth's cloud cover, observed by satellites, is strongly correlated with GCR (Svensmark et al., 1997). Clouds influence vertically integrated radiative properties of the atmosphere by both cooling through reflection of incoming shortwave radiation, and heating through trapping of outgoing longwave radiation. The net radiative impact of a particular cloud is mainly dependent upon its height above the surface and its optical thickness. High optically thin clouds tend to heat while low optically thick clouds tend to cool (Hartmann, 1993). With a current climatic estimate for the net forcing of the global cloud cover as a $17-35 \mathrm{Wm}^{-2}$ cooling, clouds play an important role in the Earth's radiation budget (Ohring and Clapp, 1980; Ramanathan et al., 1989; Ardanuy et al., 1991). Thus any significant solar influence on global cloud properties is potentially very important for Earths climate (Svensmark et al., 1997; Svensmark et al., 1998).

Figure 2 is a composite of satellite observations of Earth's total cloud cover adapted from Svensmark et al. (1997). The cloud data comprise the NIMBUS7 CMATRIX project (Stowe et al., 1988) (triangles), and secondly the International Satellite Cloud Climatology Project (ISCCP) (Rossow and Schiffer, 1991) (squares). Finally data from the Defense Satellite Meteorological Program (DMSP) 


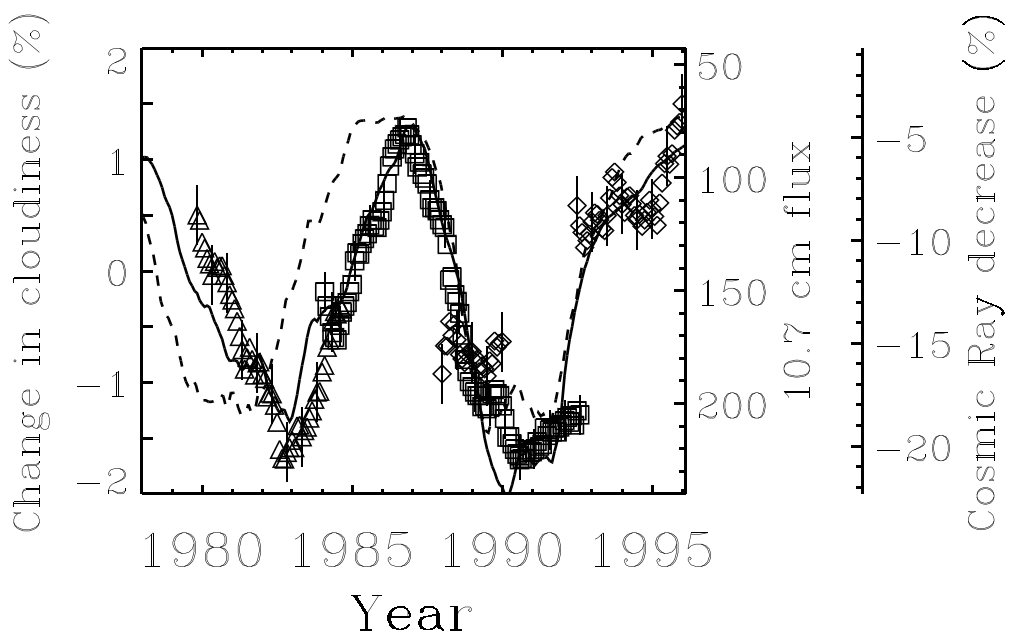

Figure 2. Composite figure showing changes in Earth's cloud cover from four satellite cloud data sets together with cosmic rays fluxes from Climax (solid curve, normalized to May 1965), and $10.7 \mathrm{~cm}$ Solar flux (broken curve, in units of $10^{-22} \mathrm{Wm}^{-2} \mathrm{~Hz}^{-1}$ ). Triangles are the Nimbus7 data, squares are the ISCCP_C2 and ISCCP_D2 data, diamonds are the DMSP data. All the displayed data have been smoothed using a 12 months running mean. The Nimbus7 and the DMSP data are total cloud cover for the Southern Hemisphere over oceans, and the ISCCP data have been derived from geostationary satellites over oceans with the tropics excluded.

Special Sensor Microwave/Imager (SSM/I) (diamonds) (Weng and Grody, 1994). Since the cloud data are obtained from four different cloud satellite programs the data presented are not homogeneous. The reason is that the instrumentation, spatial and temporal coverage is different from one satellite system to another. Therefore only the relative variations in the data can be compared. Only the most reliable data common to all satellites was used in an attempt to improve inhomogeneities. For example the DMSP satellites only retrieve data from over the oceans, therefore only cloud retrieved over oceans is used. Further, only ISCCP data retrieved from geo-stationary satellites was used due to their superior spatial and temporal coverage over the polar orbiting satellites. The tropics was excluded in the above Fig. 2 for two reasons. Due to the shielding of Earth's magnetic field there is a significant reduction of GCR flux close to the equator. Secondly, tropical cloud processes there are different compared to cloud processes at higher latitudes, e.g. the net radiative impact of clouds in the tropical regions is small compared to higher latitudes. For further details see Svensmark et al. (1997). The obtained results are not very sensitive to the selection mentioned above, and therefore the term Earth's cloud cover is used in Fig. 2.

In the figure the cloud data is compared with variation in GCR and the $10.7 \mathrm{~cm}$ radio flux from the Sun. One sees that there are clear differences between the variation of GCR and the radio flux. From 1987 to present the two follow each other. However, there is a lag between the two of almost two years prior to 1987. What is crucial in this context is that Earth's cloud cover follows the variation in 


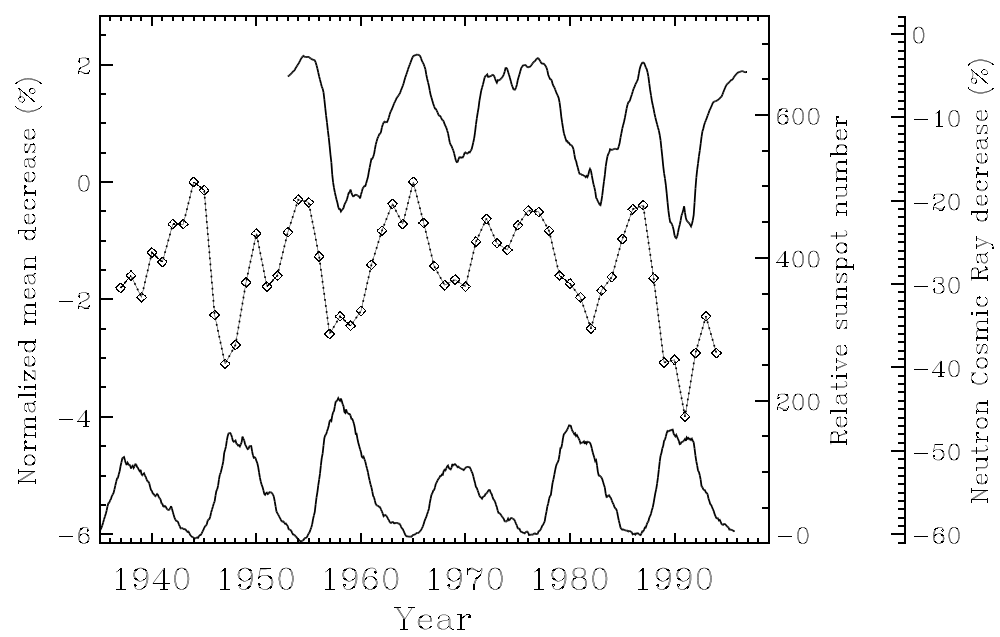

Figure 3. Top curve is cosmic ray flux from the neutron monitor in Climax, Colorado (1953-1996). Middle curve is annual mean variation in Cosmic Ray flux as measured by ionisation chambers (1937-1994). The neutron data has been normalized to May 1965, and the ionisation chamber data has been normalized to 1965 . Bottom curve is the relative sunspot number.

GCR. This is important since it indicates that it is the ionisation in the atmosphere produced by GCR that is essential in the solar climate link, and not necessarily the variations in the $10.7 \mathrm{~cm}$ flux. This radio flux follows closely variations in total solar irradiance, soft X-rays, and in ultraviolet radiation.

\subsection{INFLUENCE ON TEMPERATURE}

Having established that variations in GCR are a good candidate for indirectly influencing Earth's climate based on data covering the last solar cycle, it is important to compare variations in solar activity over a longer time span. However, there is no reliable data of cloud cover outside the period already used. But if variations in GCR cause a climatic effect it should be reflected in variations in Earth's temperature. To investigate this a long data series of GCR flux is needed. Instrumental recordings of cosmic rays started around 1935. The first measurements where performed primarily with ionisation chambers, which measure mainly the muon flux. Muons are responsible for most of the ionisation in the lower part of the troposphere (Lal et al., 1967). Ahluwalia has constructed a measure of cosmic ray flux, based on ion chambers, covering the period 1937 to 1994 (Ahluwalia, 1997), which is shown in Fig. 3. This extended data string is made by combining annually mean hourly counting rates from Cheltenham/Fredericksburg (1937-1975) and Yakutsk (1953-1994). These data represent part of the high energy GCR spectrum, and is only modulated about $4 \%$ during a solar cycle. Also contracted in Fig. 3 are 

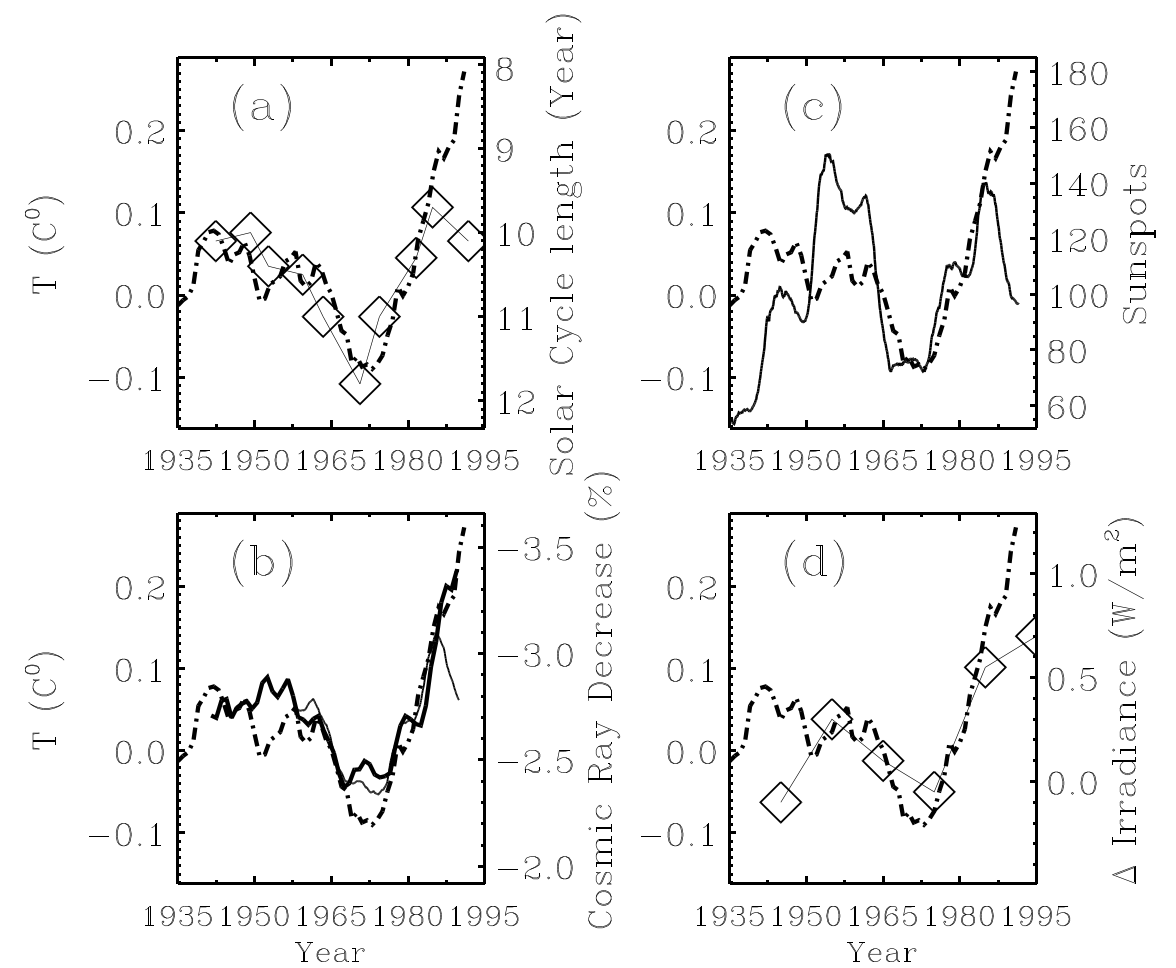

Figure 4. 11-year average of Northern Hemispheric marine and land air temperature anomalies (broken line) compared with, a) unfiltered solar cycle length. b) 11-year (box-car) average of cosmic ray flux (from ion chambers 1937-1994, normalized to 1965, thick solid line), the thin solid line is cosmic ray flux from Climax, Colorado neutron monitor (arbitrarily scale), c) 11-year (box-car) average of relative sunspot number, d) decade variation in reconstructed solar irradiance (zero level correspond to $1367 \mathrm{~W} / \mathrm{m}^{2}$, adapted from Lean et al., 1995).

data from the Climax neutron monitor (1953-1995) in Colorado, which measures the low energy nucleonic part of the GCR spectrum. For comparison the relative sunspot number is plotted, which follows closely the solar $10.7 \mathrm{~cm}$ flux. Note that even though there is a clear solar cycle modulation of the Cosmic ray flux, the minimum in GCR and are not well correlated with the maximums in sunspot number (Ahluwalia, 1996), which gives a possibility to make a distinction between long term trends in the two.

Figure 4 displays four different measures of long term solar activity together with Earth's temperature. (The temperatures used here and in the following are all temperature anomalies). In the figure, 11-year averages of the northern hemispheric land and marine temperatures (Jones, 1997) are shown in all four panels. The northern hemispheric temperatures are chosen for two reasons, first the data are superior since there are far more recordings compared to the southern hemisphere. Secondly the southern hemisphere is mainly water and the thermal inertia of the ocean tends to mask a solar forcing in contrast to the northern hemisphere. Panel 4a shows in 
addition the unfiltered solar cycle length. Panel 4b displays the 11-year averaged (ion chamber 1937-1994) cosmic ray flux (thick solid line). For comparison the Climax neutron monitor is also shown (thin solid line, scale not shown). Panel 4c shows the 11-years average of sunspot number, and finally panel $4 \mathrm{~d}$ is decade variations in reconstructed solar irradiance adapted from Lean et al. (1995). The best correspondence between solar activity and temperature seems to be between solar cycle length and variations in cosmic ray flux. However, the closest match is with the ion chamber cosmic ray data. This is interesting since these "high energy" cosmic rays are responsible for ionisation in the lowest part of the atmosphere (below about $4 \mathrm{~km}$ ), and might hint at where in the atmosphere to look for a physical effect. The variations in reconstructed solar irradiance follows more closely the variations in the sunspot number panel $4 \mathrm{~d}$.

From Fig. 4 it is seen that the temperature in the period 1970-1990 rose by approximately $0.3^{\circ} \mathrm{C}$. It is possible to compare the variation in cosmic ray flux and this temperature change via some simple assumptions. From cloud satellite observations and numerical cloud modeling it is found that a $1 \%$ change in the total composition of Earth's cloud cover correspond to $0.5 \mathrm{~W} / \mathrm{m}^{2}$ change in net radiative forcing (Rossow and Cairns, 1995). From Svensmark et al. (1997) it is known that from 1987 to 1990 global cloudiness changed approximately $3.0 \%$ which can be estimated to $1.50 \mathrm{~W} / \mathrm{m}^{2}$. In the same period cosmic rays from the ion chamber changed 3.5\% as seen in Fig. 3. We can now calculate the approximate radiative forcing by noting that the running mean 11-years average increase of cosmic rays in Fig. 4 from 1975 to 1989 is between $0.6-1.2 \%$ which then corresponds to a potential $0.3-0.5 \mathrm{~W} / \mathrm{m}^{2}$ change in cloud forcing. This is a fairly large forcing, about 2-4 times the estimated change in solar irradiance. Studies obtained from a general circulation model gave a sensitivity $\left(0.7\right.$ to $1^{\circ} \mathrm{C} / \mathrm{Wm}^{-2}$ for $\Delta S=0.25 \%$, where $S$ is the solar constant) (Rind and Overpeck, 1993). The direct influence of changes in solar irradiance is estimated to be only $0.1^{\circ} \mathrm{C}$ (Lean et al., 1995). The cloud forcing however, gives for the above sensitivity, $0.2-0.5^{\circ} \mathrm{C}$. The basic assumption in the above simple calculation is that the whole cloud volume is affected by solar activity. This is consistent with the result from Fig. 4 that shows that an increase in cloud cover results in lower temperatures. Solar forcing therefore has the potential to explain a significant part of the temperature changes over the period studied.

\subsection{MAUnder Minimum}

The Maunder minimum (MM) (1645-1715) is a famous period in the Sun's history. It is a period where very few sunspots where observed. In 1976 Eddy suggested that during this period the solar output was lower, and that this reduction could explain the extreme climatic conditions (Eddy, 1976) at the time. Since then various efforts have gone into reconstructing solar irradiance back in time. One of the better measures of solar activity is the sunspot number, which is known over the last 400 years. Therefore it is tempting to look for a relation between sunspot number and changes in solar irradiance observed by satellites during the last 20 
years, since such a relation can be used in an attempt to reconstruct solar irradiance back in time. Figure 5a shows part of a reconstructed solar irradiance curve constructed by Lean et al. (Lean et al., 1995), centered around the MM. Assuming that the sun is in a non-cyclic state during the MM, the irradiance is found to be lower by $0.24 \%\left(\approx 0.82 \mathrm{~W} / \mathrm{m}^{2}\right.$ when averaged over Earth's surface) lower than the present day value (Lean et al., 1992). As a result, the reconstructed solar irradiance shown in Fig. 5a is nearly constant during the whole period of the MM. Figure $5 \mathrm{~b}$ shows the variations in ${ }^{10} \mathrm{Be}$, and is a signature of changes in cosmic ray flux during the MM (Beer et $a l ., 1991)$. The ${ }^{10} \mathrm{Be}$ data thereby contains information on variations in the solar wind magnetic activity. It is seen that there seem to be a cyclic magnetic behavior through the MM (Beer et al., 1985), and that the very low solar magnetic activity is occurring at the end of the MM, i.e. 1690-1715. It is interesting to compare the above curves for solar irradiance and the ${ }^{10} \mathrm{Be}$ data, i.e. Figs. 5a and $\mathrm{b}$ with a recently reconstructed temperature curve, Fig. 5c, for the northern hemisphere (Jones et al., 1998). A striking similarity between the ${ }^{10} \mathrm{Be}$ curve and the temperature is seen. In fact the decade $1690-1700$ is the coldest during the last 1000 years, at the same time as the ${ }^{10} \mathrm{Be}$ concentration has the largest peak. Assuming there is a solar impact on climate during this period, it seems less likely climate was affected by the Sun in a non-cyclic state, which gives the nearly constant
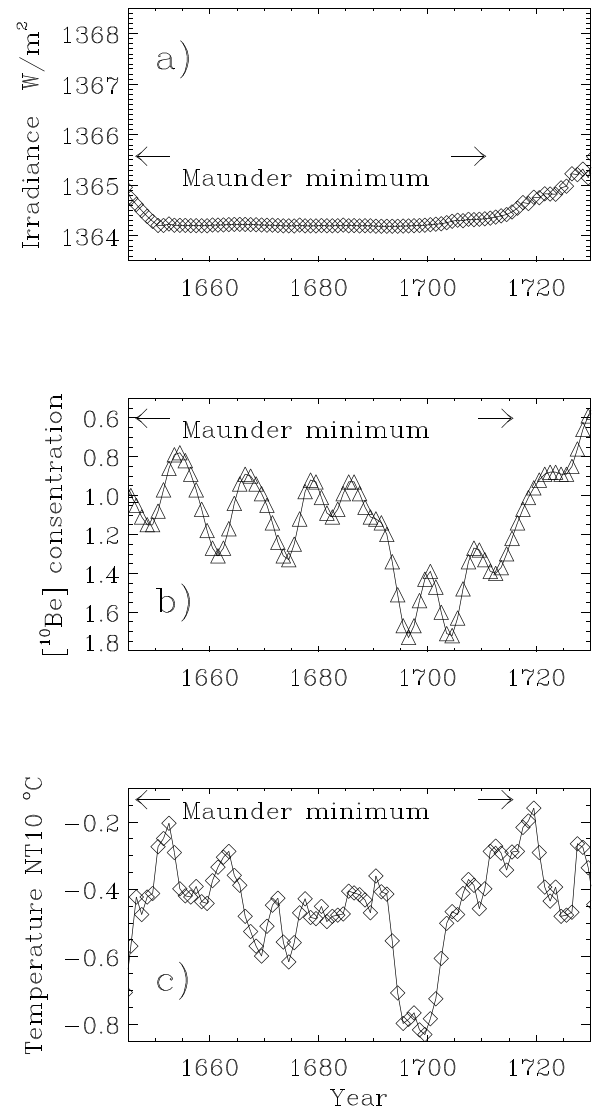

Figure 5. Panel a): Variation in in reconstructed solar irradiance during the Maunder minimum, from Lean et al. Panel b): Variation in ${ }^{10} \mathrm{Be}$ concentration during the Maunder minimum (note that the ${ }^{10} \mathrm{Be}$ axis is reversed). Panel c): Reconstructed temperature anomalies of the northern hemisphere during the Maunder minimum. solar irradiance, according to the model of Lean et al. Rather, the good agreement between temperature and ${ }^{10} \mathrm{Be}$ concentration could suggest that cosmic rays are important in a sun/climate link. 
Alternatively it could be that the origin of the irradiance changes during the MM are not understood. Of course the above conclusions hinge on how accurate the data are. The peak in the ${ }^{10} \mathrm{Be}$ curve might in fact be broader (Beer, 2000), and the true shape of the minimum in the temperature curve could be different. However reconstructed temperatures of the northern hemisphere, by Mann et al. (1998), also finds that the coldest decade is $1690-1700$.

\section{Conclusions}

Climate has been varying during all time. The origin of these variations has previously, and almost exclusively, been attributed to internal causes. For example volcanic dust in the stratosphere can cause cooling of the order $0.5^{\circ} \mathrm{C}$ for a year or more. The same goes for the atmospheric/ocean oscillation in the Pacific called El Niño Southern Oscillation . So it is expected that the annual variations in temperature will be a composite of several causes, of which only one is a solar influence. However, at time scales greater than 10 years it looks like the Sun has a significant influence on climate variations. This statement is based on the qualitative agreement between isotopes and proxy data for Earth's temperature over the last 1000 years.

A remarkable correlation between cosmic ray flux and variations in Earths cloud cover has been demonstrated. Since clouds are important for the Earths energy balance, a solar influence on clouds could be the main cause for the observed correlations between the sun and Earths climate. However, as is well known a good correlation does not guarantee a physical cause and effect. It is therefore necessary to get an understanding of the microphysical mechanism that connects solar activity with Earth's cloud cover. If the influence of cosmic rays on clouds is real, then it is thought that ionisation produced by GCR affects the microphysics in cloud formation. There is currently an initiative do an experiment at European centre for particle physics CERN, in Geneva, to test this hypothesis. The idea is to study the effect of ionisation caused by cosmic rays on droplet formation. This will be done in a cloud chamber with a high degree of control of all relevant parameters. However, future efforts to understand the importance of solar activity must also involve observations. In particular it is important to find what type of clouds are affected. This can be done on the one hand from further studies by satellite observation, but also by regional observations of cloud/aerosol formation, e.g. by LIDAR. It is hoped that the present study might increase the interest in finding a physical mechanism.

\section{Acknowledgements}

I thank J.van der Plicht for providing the C14 data that Fig. 1 was based on. Also thanks to J. Beer for providing ${ }^{10} \mathrm{Be}$ data that Fig. $5 \mathrm{~b}$ was based on. 


\section{References}

Ahluwalia, H. S., and Wilson, M.D.: 1996, 'Present Status of the Recovery Phase of Cosmic ray 11-year Modulation', J. Geophys. Res. 101, 4879-4883.

Ahluwalia, H. S.: 1997, 'Galactic Cosmic Ray Intensity Variations at High Latitude sea Level Site 1937-1994', J. Geophys. Res. 102, 24,229-24,236.

Ardanuy, P., Stowe, L. L., Gruber, A., and Weiss, M.: 1991, 'Shortwave, Longwave, and net CloudRadiative Forcing as Determined From Nimbus-7 Observations', J. Geophys. Res. 96, 1-2.

Beer, J., et al.: 1985, 'Accelerator Measurements of 10Be: The 11 Year Solar Cycle From 1180-1800', Nucl. Instru. Meth. Phys. Res. B10/11, 415-418.

Beer, J., Raisbeck, G. M., and Yiou, F.: 1991, in C. P. Sonett, M.S. Giampapa, and M.S. Matthews (eds.), The Sun in Time, University of Arizona Press.

Beer, J., personal communication.

Dickinson, R., 1975, 'Solar Variability and the Lower Atmosphere', Bull. Am. Met. Soc. 56, 12401248.

Eddy, J. A.: 1976, ‘The Maunder Minimum', Science 192, 1189-1202.

See for example the book: Herman, J.R., and Goldberg, R. A.: 1978, Sun, Weather, and Climate, NASA SP 426.

Friis-Christensen, E., and Lassen, K.: 1991, 'Length of the Solar Cycle: An Indicator Closely Associated With Climate', Science 254, 698-700.

Haigh, J. D.: 1996, 'The Impact of Solar Variability on Climate', Science 272, 981-984.

Hartmann, D. L.: 1993, 'Radiative Effects of Clouds on Climate', in P. V. Hobbs (ed.), AerosolCloud-Climate Interactions, Academic Press, pp. 151-173.

Jones, P. D.: 1997, 'Hemispheric and Global Temperatures, 1851-199, Part 1', Climate Monitor 25, 20-30; Jones, P. D.: 1997, 'Hemispheric and Global Temperatures, 1851-1996, Part 2', Climate Monitor 25, 66-77.

Jones, P.D., Briffa, K. R., Barnett, T. P., and Tett, S.F.B.: 1998, 'High-Resolution Palaeoclimatic Records for the Last Millennium: Interpretation, Integration and Comparison With General Circulation Model Control-run Temperatures', The Holocene 8, 455-471.

Labitzke, K., and van Loon, H.: 1993, 'Some Recent Studies of Probable Connections Between Solar and Atmospheric Variability', Ann. Geophysicae 11, 1084-1094.

Lal, D., and Peters, B.: 1967, in S. Flugge (ed.), Encyclopedia of Physics, number XLVI in 2, Springer-Verlag, Berlin Heidelberg, p. 551.

Lassen, K., and Friis-Christensen, E.: 'Variability of the Solar Cycle Length During the Last Five Centuries and the Apparent Association with Terrestrial Climate', J. atm. terr. Phys. 57, 835-845.

Lean, J., Skumanich, A., White, O.: 1992, 'Estimating the Suns Radiative Output During the Maunder Minimum', Geophys. Res. Lett. 19, 1591-1594.

Lean, J., Beer, J., and Breadley, R.: 1995, 'Reconstruction of Solar Irradiance Since 1610: Implications for Climate Change', Geophys. Res. Lett. 22, 3,195-3,198.

Mann, M.E., Bradley, R.S., and Huges, M.K.: 1998, 'Global-Scale Temperature Patterns and Climate Forcing Over the Past six Centuries', Nature 392, 779-787.

Ney, E. R.: 1959, 'Cosmic Radiation and the Weather', Nature 183, 451-452.

Ohring, G., and Clapp, P. F.: 1980, 'The Effect of Changes in Cloud Amount on the net Radiation at the top of the Atmosphere', J. Atmos. Sci. 37, 447-457.

Pudovkin, M.I., and Raspopov, O. M.: 1992, 'The Mechanism of Action of Solar Activity on the State of the Lower Atmosphere and Metorological Parameters (A Review)', Geomagn. and Aeronomy 32, 593-608.

Pudovkin, M., and Veretenenko, S.: 1995, 'Cloudiness decrease associated with Forbush Decreases of Galatic Cosmic Rays’, J. Atmos. Terr. Phys. 57, 1349-1355. 
Ramanathan, Cess, R. D., Harrison, E. F., Minnis, P., Barkstrom, B. R., Ahmad, E., and Hartmann, D.: 1989, 'Cloud-radiative Forcing and Climate: Results From the Earth's Radiation Budget Experiment', Science 243, 57-63.

Rind, D., and Overpeck, J.: 1993, 'Hypothesized Causes of Decade-to-Century Climate Variations', Quat. Sci. Rev. 12, 357-374.

Rossow, W. B., and Schiffer, R.: 1991, 'International Satellite Cloud Climatology Project (ISCCP) cloud data products', Bull. Amer. Meteor. 72, 2-20.

Rossow, W. B., and Cairns, B.: 1995, 'Monitoring changes of clouds', J. Climate 31, 305-347.

Shindell, D., Rind, D., Balabhandran, N., Lean, J., and Lonergan, P.: 1999, 'Solar Cycle Variability, Ozone and Climate', Science 284, 305.

Stowe, L. L., Wellemayer, C. G., Eck, T. F., Yeh, H.Y.M., and the Nimbus-7 Team: 1988, 'Nimbus-7 global cloud climatology, Part 1: Algorithms and validations', J. Climate 1, 445-470.

Svensmark, H., and Friis-Christensen, E.: 1997, 'Variation of cosmic ray flux and global cloud coverage - a missing link in solar-climate relationships', J. Atm. Sol. Terr. Phys. 59, 1225-1232.

Svensmark, H.: 1998, 'Influence of cosmic rays on Earth's climate', Phys. Rev. Lett. 81, 5027-5030.

Tinsley, B. A.: 1996, 'Solar Wind Modulation of the Global Electric Circuit and the Apparent Effects on Cloud Microphysics, Latent Heat Release, and Tropospheric Dynamics', J. Geomag. Geoelectr. 48, 165-175.

Weng, F., and Grody, N.C.: 1994, 'Retrieval of Cloud Liquid Water Using the Special Sensor Microwave Imager', J. Geophys. Res. 99, 25,535; Ferraro, R. R., Weng, F., Grody, N. C., and Basist, A.: 1996, 'An Eight-Year (1987-1994) Time Series of Rainfall, Clouds, Water Vapor, Snow Cover, and Sea Ice Derived From SSM/I Measurements', Bull. Am. Met. Soc. 77, 891-905.

Address for Offprints: Henrik Svensmark, Danish Space Research Institute, Juliane Maries Vej 30,

DK-2100 Copenhagen $\emptyset$, Denmark; hsv@dsri.dk 
new2_sven0606.tex; ; ; p.12 